\title{
Macrophage Polarization Contributes to the Anti-Tumoral Efficacy of Mesoporous Nanovectors Loaded with Albumin-Bound Paclitaxel
}

\author{
Fransisca Leonard ${ }^{1}$, Louis T. Curtis ${ }^{2}$, Matthew James Ware ${ }^{3}$, Taraz Nosrat ${ }^{1}$, Xuewu Liu ${ }^{1}$, \\ Kenji Yokoi ${ }^{1}$, Hermann B. Frieboes ${ }^{2,4 t}$ and Biana Godin ${ }^{1+t}$ \\ ${ }^{1}$ Department of Nanomedicine, Houston Methodist Research Institute, Houston, TX, United States, ${ }^{2}$ Department of \\ Bioengineering, University of Louisville, Louisville, KY, United States, ${ }^{3}$ Department of Surgery, Baylor College of Medicine, \\ Houston, TX, United States, ${ }^{4}$ James Graham Brown Cancer Center, University of Louisville, Louisville, KY, United States
}

Therapies targeted to the immune system, such as immunotherapy, are currently shaping a new, rapidly developing branch of promising cancer treatments, offering the potential to change the prognosis of previously non-responding patients. Macrophages comprise the most abundant population of immune cells in the tumor microenvironment (TME) and can undergo differentiation into functional phenotypes depending on the local tissue environment. Based on these functional phenotypes, tumor-associated macrophages (TAMs) can either aid tumor progression (M2 phenotype) or inhibit it (M1 phenotype). Presence of M2 macrophages and a high ratio of M2/M1 macrophages in the TME are clinically associated with poor prognosis in many types of cancers. Herein, we evaluate the effect of macrophage phenotype on the transport and anti-cancer efficacy of albumin-bound paclitaxel (nAb-PTX) loaded into porous silicon multistage nanovectors (MSV). Studies in a coculture of breast cancer cells (3D-spheroid) with macrophages and in vivo models were conducted to evaluate the therapeutic efficacy of MSV-nAb-PTX as a function of macrophage phenotype. Association with MSV increased drug accumulation within the macrophages and the tumor spheroids, shifting the inflammation state of the TME toward the pro-inflammatory, anti-tumorigenic milieu. Additionally, the treatment increased macrophage motility toward cancer cells, promoting the active transport of therapeutic nanovectors into the tumor lesion. Consequently, apoptosis of cancer cells was increased and proliferation decreased in the MSV-nAb-PTX-treated group as compared to controls. The results also confirmed that the tested system shifts the macrophage differentiation toward an M1 phenotype, possessing an anti-proliferative effect toward the breast cancer cells. These factors were further incorporated into a mathematical model to help analyze the synergistic effect of the macrophage polarization state on the efficacy of MSV-nAb-PTX in alleviating hypovascularized tumor lesions. In conclusion, the ability of MSV-nAb-PTX to polarize TAM to the M1 phenotype, causing (1) enhanced penetration of the drug-carrying macrophages to the center of the tumor lesion and (2) increased toxicity to tumor cells may explain the increased anti-cancer efficacy of the system in comparison to nAb-PTX and other controls.

Keywords: macrophage polarization, nanotherapy, breast cancer, computational modeling, tumor microenvironment 


\section{INTRODUCTION}

Tumor initiation, growth, and progression rely on the bidirectional interaction of the tumor cells with the cells in the tumor microenvironment (TME). Solid tumors comprise variable amounts of neoplastic and stromal cells. The tumor stroma includes endothelial cells, fibroblasts, and immune cells, mainly macrophages and lymphocytes. Macrophages are a plastic and heterogeneous immune cell population. In particular, tumor-associated macrophages (TAMs), derived from monocytic precursors, comprise the most abundant population of immune cells in the TME (1-3). Macrophages in the TME can undergo functional changes and be polarized from the resting M0 phenotype to the classically activated pro-inflammatory M1 or anti-inflammatory (alternatively activated) M2 general subsets, based on the stimuli in the residing milieu (4). M1 macrophages are characterized by their expression of inducible nitric oxide synthase, production of pro-inflammatory cytokines (e.g., TNF, IL-1, -6, and -12) and reactive oxygen species (ROS). This subpopulation of macrophages promotes strong immune responses and is antitumorigenic $(5,6)$. On the contrary, M2 macrophages antagonize the inflammation and are present in the advanced stages of the healing process. M2 macrophages enhance the formation of tumor stroma by recruiting fibroblasts and activating their differentiation to myofibroblasts, causing the release of pro-angiogenic factors that enable recruitment of endothelial progenitor cells and neovasculogenesis and suppression of inflammation through decreased production of ROS and pro-inflammatory cytokines $(7,8)$. While M2 macrophages possess a significant role in host defense and Th2-mediated activation of the humoral immune response, their presence in the TME promotes tumor development. Presence of $\mathrm{M} 2$ macrophages and a high ratio of M2/M1 macrophages in the TME are clinically associated with poor prognosis in many types of cancers (9-12).

It is noteworthy that the tight distinction between M1 and M2 macrophages does not fully describe the continuum of their functions and can be considered as a simplified classification of the two sides of the polarization spectrum (13). TAMs are usually considered M2-like macrophages (14-16), which abandon the M1-related innate and adaptive immune responses capable of destroying malignant cells. Changes in the stimuli of the TME can cause reprogramming of macrophages from an M1 phenotype to an M2-activated state and vice versa $(17,18)$. Macrophage reprogramming has been recently shown to inhibit cancer progression and metastasis $(19,20)$. Controlling the macrophage polarization state in the TME could provide a novel approach to treating related diseases. Reprogramming M2 macrophages toward the M1 subset is an important focus of recent research, with a number of recent publications demonstrating the ability of some nanomaterials to induce macrophages between polarization states (21-23).

Our previous studies have shown that TAMs play a significant role in therapeutic efficacy of albumin-bound paclitaxel (nAbPTX) loaded into porous silicon multistage nanovectors (MSV) in liver metastasis of breast and lung tumors (24). Although tumor lesions in the liver have inefficient vascularization, we demonstrated an increased concentration of macrophages acting as chemotherapeutic depots near these lesions. This significantly enhanced efficacy and extended survival in two tested animal models of liver metastases. Furthermore, we have mathematically modeled the efficacy of MSV-nAb-PTX nanovectors in 3D tumor models to project MSV-nAb-PTX efficacy in hypovascularized lesions and concluded that the proposed $3 \mathrm{D}$ coculture of macrophages and tumor cells serve as a good model for the in vivo condition (25). However, based on the integrated experimental and mathematical analysis of the data, it appears that the efficacy of MSV-nAb-PTX was more than expected solely from macrophages acting as a depot for the drug.

Herein, we aim to evaluate the effect of macrophage phenotype on the anti-cancer efficacy of MSV-nAb-PTX, as well as the effect of these nanovectors on macrophage polarization state. For this purpose, the experiments were performed in vitro using a validated coculture of breast cancer tumor cells (3D spheroids) with macrophages and in vivo in the breast cancer tumor metastasis mouse model. Our in vitro and in vivo findings show that treatment with MSV-nAb-PTX affected the macrophages to polarize from the M2-type to the anti-tumorigenic M1 phenotype. Additionally, the treatment increased macrophage motility toward cancer cells, promoting the penetration of therapeutic nanovectors into the tumor lesion. These findings were further incorporated into a mathematical model to help analyze the synergistic effect of macrophage polarization state on the efficacy of MSV-nAb-PTX in treating hypovascularized tumor lesions.

\section{RESULTS}

\section{Efficacy of Macrophage-Associated MSV-nAb-PTX in 3D TME In Vitro Model of Hypovascularized Breast Tumor Lesions}

In this study, we use a validated TME model of hypovascularized breast tumor lesions, which consist of macrophages surrounding 4T1 cell spheroids. Rapamycin was used as a factor shifting polarization of macrophages toward the M1 phenotype (26), a positive control of macrophage differentiation.

As shown in Figure 1, Ki67 staining indicated that the cells in the control spheres actively proliferated. All treatment groups including nAb-PTX, MSV-nAb-PTX, and rapamycin induced apoptosis in the spheres [terminal deoxynucleotidyl transferase dUTP nick end labeling (TUNEL) staining] and reduced tumor cell proliferation (Figure 1A). Similar to previously reported in vivo data (24), treatment with MSV-nAb-PTX and nAb-PTX both resulted in a high apoptosis rate, as shown by green signals from the cells in Figure 1A. Rapamycin induced apoptosis in a similar rate to MSV-nAb-PTX, and cell proliferation was only slightly inhibited by rapamycin. This inhibition was not as efficient as exhibited in the nAb-PTX and MSV-nAb-PTX treatment groups. Spheroids treated with nAb-PTX displayed low proliferation profiles, as observed by a weak Ki67 signal, mostly within the $\sim 75 \mu \mathrm{m}$ of the outer layer of the spheres. In the MSVnAb-PTX-treated group, the effect was more pronounced and only cells within $\sim 20 \mu \mathrm{m}$ from the outer layer of the spheroids were still proliferating (Figure 1A). The ratio of the tumor cells 


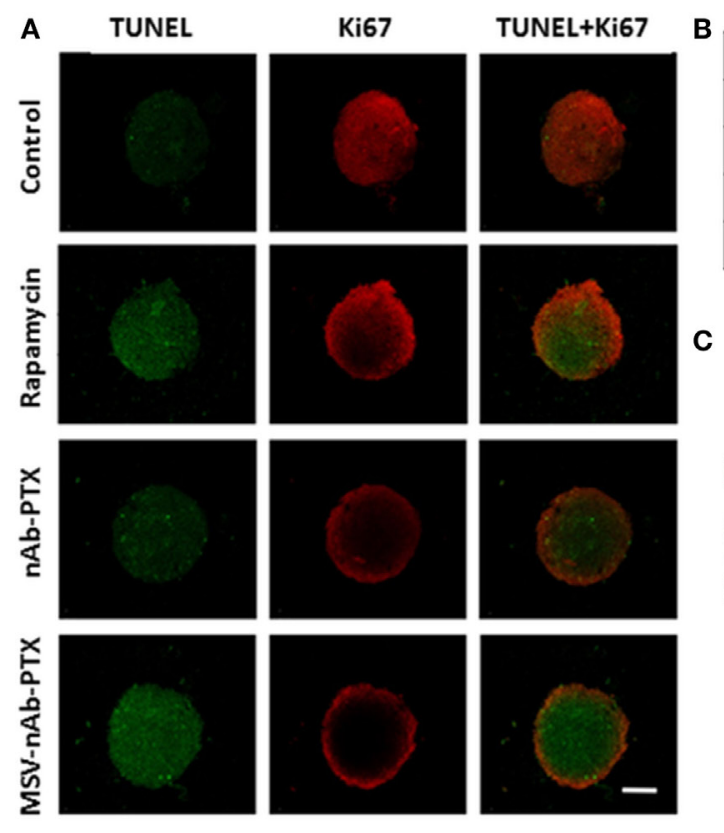

\begin{tabular}{|l|c|}
\hline & Ratio apoptosis/proliferation \\
\hline Control & $0.42 \pm 0.14$ \\
\hline Rapamycin & $3.41 \pm 1.15$ \\
\hline nAb-PTX & $1.74 \pm 1.02$ \\
\hline MSV-nAb-PTX & $5.57 \pm 2.37$ \\
\hline
\end{tabular}

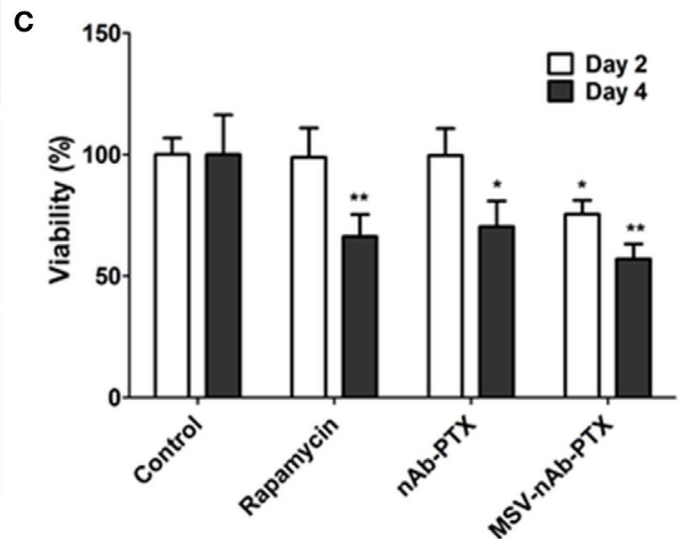

FIGURE 1 | Therapeutic efficacy of the systems in 3D breast tumor tumor microenvironment model. (A) Confocal microscopy images from a coculture of 4T1 cancer spheroids and macrophages pretreated with multistage nanovectors (MSV)-albumin-bound paclitaxel (nAB-PTX), nAb-PTX, and rapamycin. Evaluation of apoptosis (terminal deoxynucleotidyl transferase dUTP nick end labeling, green signals) and cell proliferation (Ki67, red signals). (B) Ratio of apoptotic/proliferating cell signals as quantified by image analysis $(n=6)$. (C) Viability of the breast cancer cells as measured by 3-(4,5-dimethylthiazol-2-yl)-2,5-diphenyltetrazolium bromide assay at 48 and $96 \mathrm{~h}$ and normalized to untreated control. Mean $\pm \mathrm{SD}(n=9),{ }^{\star} p<0.05$; ${ }^{\star \star} p<0.01 \mathrm{compared}$ to control.

undergoing apoptosis/proliferation is in the following order: MSX-nAb-PTX > rapamycin $>$ nAb-PTX $>$ untreated control (Figure 1B). Furthermore, 3-(4,5-dimethylthiazol-2-yl)-2,5-diphenyltetrazolium bromide (MTT) assay showed that at 2 days from treatment, tumor cell viability was reduced (by $>30 \%$ ) only in the cells treated with MSV-nAb-PTX. At 4 days, more than $30 \%$ of tumor cells were not viable following preincubation of macrophages with nAb-PTX and rapamycin, while MSV-nAbPTX reduced viability by $>60 \%$ (Figure 1C).

\section{Macrophage Pretreatment with MSV-nAb- PTX Shifts Their Phenotype toward M1}

To investigate the effect of the systems on macrophage polarization state, macrophages pretreated with MSV-nAb-PTX, nAbPTX, and rapamycin and incubated with breast tumor spheres were tested vs. untreated control for the expression of the cell surface markers CD80 and CD204 (markers for M1 and M2 general polarization states, respectively) (Figure 2). Untreated macrophages in coculture of tumor spheres displayed the M2-like phenotype, as indicated by $>85 \%$ of the population positive to CD204 staining (Figures 2A,B). This finding is in line with the general polarization of TAM toward the M2 phenotype, as documented previously (5). More than $96 \%$ of macrophages in coculture shifted to an M1-like phenotype (CD80 expression) following the treatment with MSV-nAb-PTX. In nAb-PTX- and rapamycin-treated systems, $44.0 \pm 9.6$ and $65.6 \pm 10.1 \%$ of cells expressed M1 membrane marker.
We have further confirmed these findings in vivo (Figure 2C) in the mouse model of liver metastasis of breast tumors. The predominant population of macrophages in the untreated control group was of M2-like polarization state. MSV-nAb-PTX significantly shifted the population of macrophages toward the M1 phenotype (by twofold), while nAb-PTX had no effect on the macrophage polarization state (Figure 2D). Interestingly, more macrophages were present in the breast cancer metastatic liver lesions treated with MSV-nAb-PTX, which prompted us to look for the effect of nanovectors on macrophage migration.

\section{Effect of MSV-nAb-PTX on Macrophage Migration toward and into 4T1 Cancer Cell Spheres}

In order to evaluate the effect of MSV-nAb-PTX pretreatment on macrophage migration toward the tumor spheres and into the sphere core, experiments were performed in the $3 \mathrm{D}$ TME model we previously developed (25). Time-lapse videos of livecell images of pretreated macrophages introduced to the tumor spheroids have shown specific directionality and enhanced speed of macrophages pretreated with MSV-nAb-PTX as compared to controls (Figure 3). NIS elements analysis of the videos revealed an increased speed of macrophages treated with MSV-nAb-PTX within the first $5 \mathrm{~h}$ (Figure 3B). The increased speed does correlate with a slight increase in path length of the distance traveled by MSV-nAb-PTX macrophages (Figure 3C). All other treatments did not alter the path length compared to the control. However, 

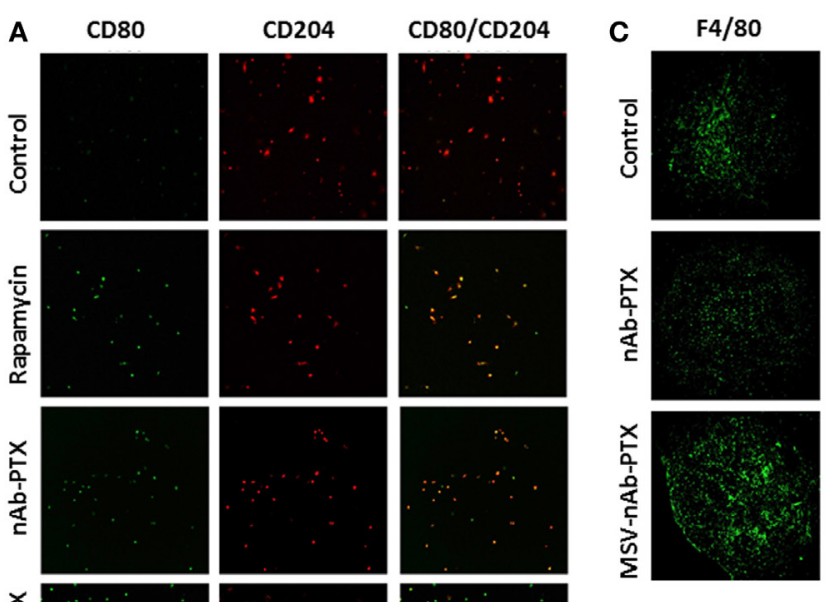

CD204

F4/80/CD204
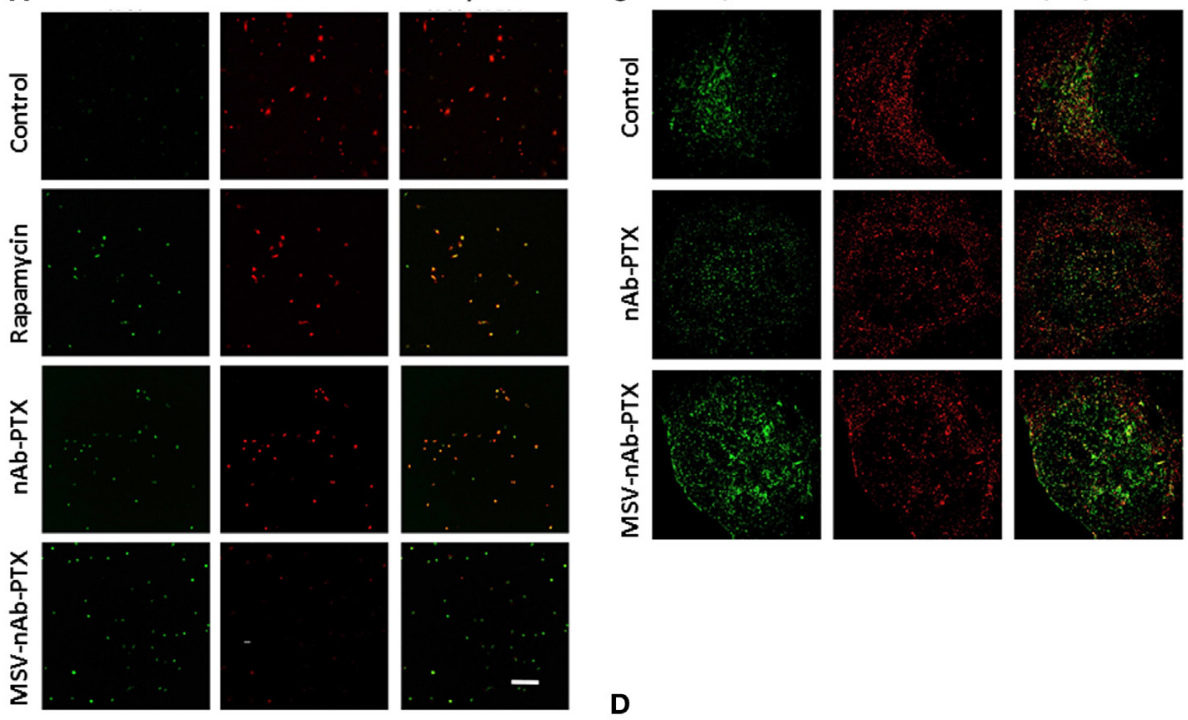

B
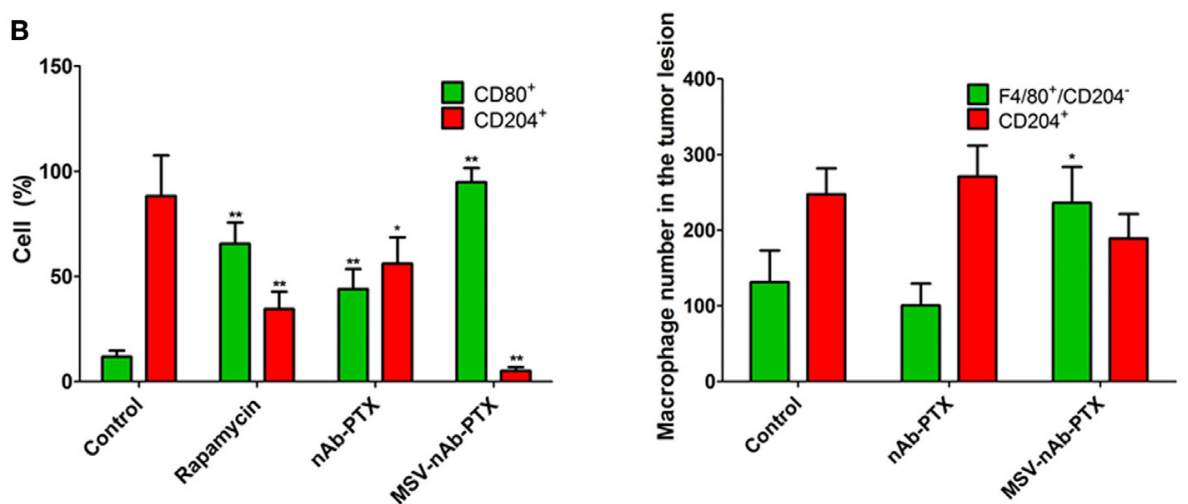

FIGURE 2 | Polarization of macrophages in response to treatment in vitro and in vivo. (A) Confocal microscopy images from a coculture of 4T1 cancer spheroids and macrophages pretreated with multistage nanovectors (MSV)-albumin-bound paclitaxel (nAB-PTX), nAb-PTX, and rapamycin. Macrophages are immunostained for either CD80 (green, M1 marker) or CD204 (red, M2 marker) membranal expression. (B). Quantification of CD80 and CD204 signals from the images presented in (A); (C) confocal images of breast tumor lesions in the liver stained for F4/80 (green, total macrophages) and CD204 (red, M2 macrophages). (D) Quantification of M1 and M2 signals (M1 number obtained from F4/80+/CD204- cells). The results are presented as mean \pm SD $(n=6-9)$, ${ }^{*} p<0.05 ;{ }^{* *} p<0.01$ to control.

the most significant change was observed in the directionality of the macrophage migration. The analysis of macrophage displacement toward the tumor sphere within the $5 \mathrm{~h}$ time frame showed a significantly specific movement toward the tumor spheres by the macrophages treated with MSV-nAb-PTX. On the other hand, no specific directionality in the movement of macrophages was observed in the cells treated with MSV or nAb-PTX (Figure 3D).

Macrophages pretreated with various systems were tracked and counted in the different depths of the tumor sphere, in increments of $50 \mu \mathrm{m}$ (Figure 4), focusing on the central part of the spheroid (average diameter $450-500 \mu \mathrm{m}$ ). The density of macrophages in the deep layers of the tumor sphere significantly increased (>2-fold compared to control) after they were pretreated with MSVnAb-PTX treatment. nAb-PTX only caused moderate increase in the macrophage number in the innermost layer of the spheres. Further analysis revealed that most of the macrophages found in the center of the spheres were M1-like phenotype. These data correlate well with an in vivo analysis of macrophage localization in breast cancer liver metastatic lesions previously published (25).

We further tested various components of the MSV-nAb-PTX system to determine the factors crucial for macrophage motility toward the center of the sphere (Figure 4C). Various elements of MSV-nAb-PTX were tested for their effect on macrophage motility: fluorescently labeled albumin (Ab) as a major component of nAb-PTX; MSV; MSV-Ab; and nAb-PTX. MSV did not affect the number of macrophages in the center of the tumor spheroid as compared to untreated control, while $\mathrm{Ab}, \mathrm{MSV}-\mathrm{Ab}$, and $\mathrm{nAb}$ PTX, surprisingly, slightly increased it. MSV-nAb-PTX enabled an increased migration of the macrophages into the deep layers of the tumor sphere. The number of macrophages in the deep layers of the tumor sphere treated with MSV-nAb-PTX was more than the summary of the effects of all individual components of the system, pointing toward the potential synergy of the factors being involved. 

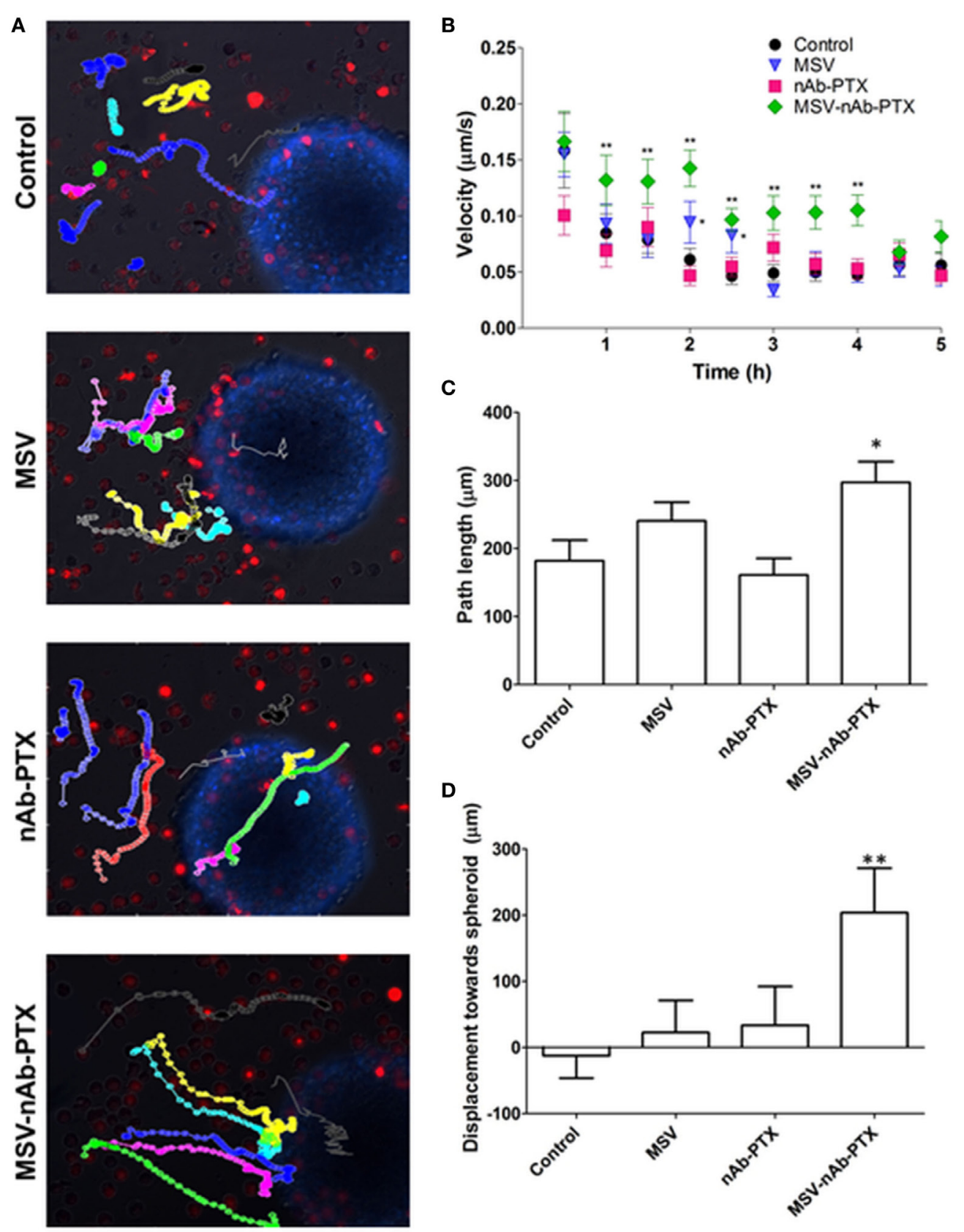

FIGURE 3 | Tracking of macrophage migration kinetics, directionality, and dynamics as a function of treatments. (A) Macrophage trajectories following the preincubation with multistage nanovectors (MSV)-albumin-bound paclitaxel (nAb-PTX), nAb-PTX, MSV, and no treatment control were tracked relative to the movement of the tumor spheres. Individual trajectories are presented in different colors. Red: macrophages stained DiD membrane dye. Blue: 4T1 breast tumor cells stained with Hoechst 33342 nucleus dye. The trajectories were tracked by live imaging for $5 \mathrm{~h}$ and analyzed for velocity (B), path length (C), and directionality (D) based on the displacement toward the breast tumor. The results are presented as mean \pm SEM $(n=20-25),{ }^{*} p<0.05 ;{ }^{* *} p<0.01$ as compared to control.

\section{Effect of MSV-nAb-PTX Pretreatment of Macrophages on Cytokine Production by the Tumors In Vitro and In Vivo}

The main function of the macrophages in the TME is tightly related to their interaction with cancer cells, resulting in the secretion of soluble factors that shape the tumor milieu. Therefore, we further performed a thorough analysis of the cytokines and chemokines in the TME 3D model in vitro and in hepatic metastases of cancerous breast lesions in vivo. Interestingly, neither $\mathrm{nAb}-\mathrm{PTX}$ nor MSV-nAb-PTX had an effect on the release of the cytokines from the macrophages following direct incubation with the systems (Figure S1 in Supplementary Material). The quantification of the factors released by the tumor cells as a response to the conditioned 


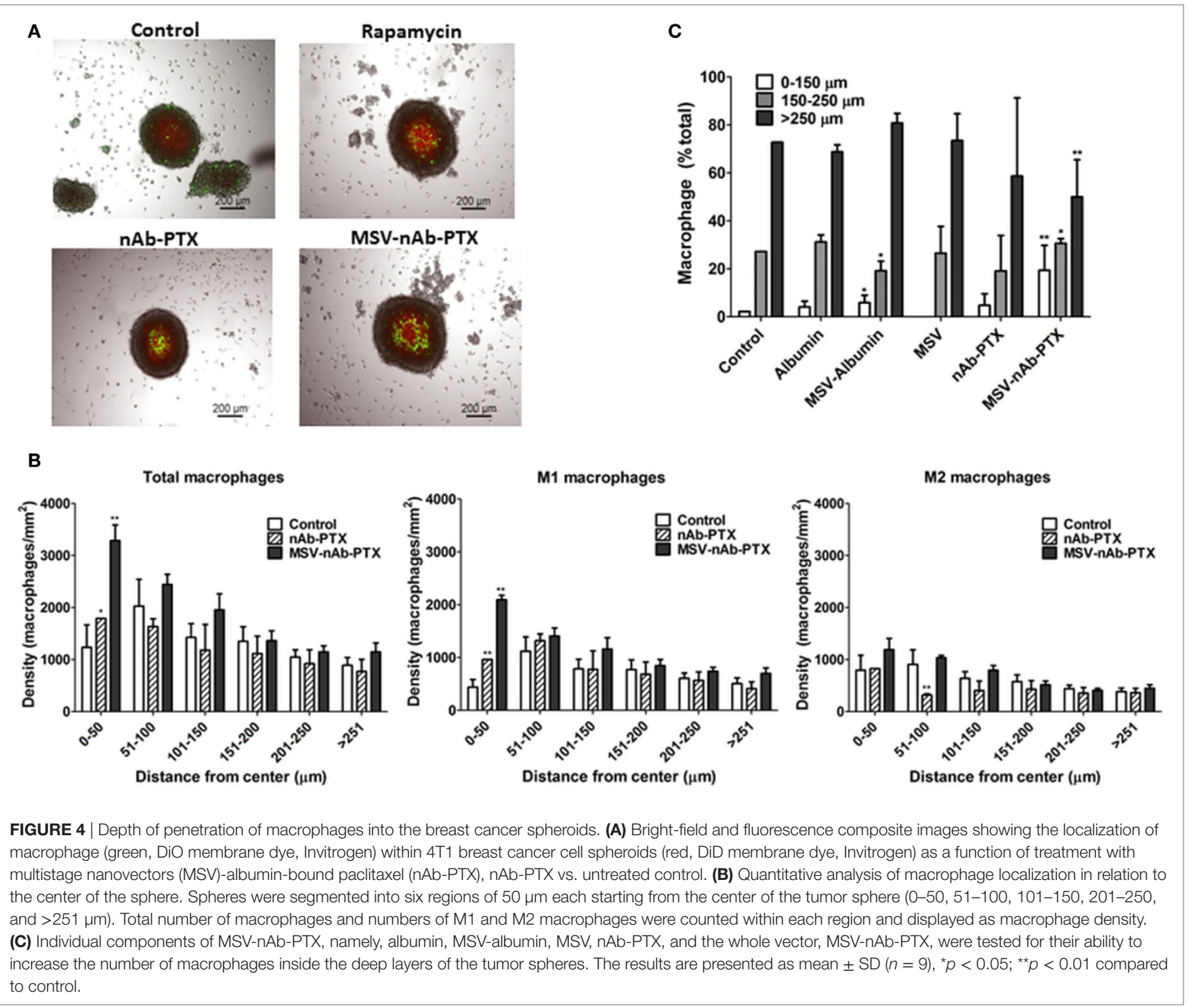

media from macrophages pretreated with nAb-PTX and MSV$\mathrm{nAb}-\mathrm{PTX}$ in vitro and in vivo is summarized in Figure 5. Cytokine levels measured in tumor cells in response to conditioned media and in vivo follow very similar trends. The following factors were increased in both in vitro and in vivo settings in the TME for MSV-nAb-PTX: G- colony-stimulating factor (CSF), GM-CSF, IFN-gamma, IL-1beta, IL-6, IL-12, IP-10, CCL3, CCL4, CCL5, and TNF-alpha. These data point toward the significant effect of the whole MSV-nAb-PTX system on the macrophage polarization state in vitro and in vivo. Interestingly, only MCP-1 increased for $\mathrm{nAb}$-PTX-treated macrophages both in vitro and in vivo settings.

\section{Mathematical Modeling to Simulate Effect of Macrophage Polarization on Tumor Response In Vivo}

In order to further analyze the treatment efficacy of MSV-nAbPTX, we mathematically modeled the effect of MSV-nAb-PTX on hypovascularized liver lesions in vivo coupled with macrophage differentiation into M1 and M2 subtypes. As in our previous work (25), the lesion growth was simulated in parallel with the dynamic drug distribution.

Figure 6 illustrates the effects of therapy with the MSVnAb-PTX-loaded macrophages. Undifferentiated macrophages extravasate from the vasculature and migrate toward the lesion based on the chemotactic gradient of attractants (such as proangiogenic factors released by tumor cells) in the surrounding microenvironment. During this process, the macrophages differentiate into M1 or M2 subtypes depending on the ratio of pro-M1 and pro-M2 macrophage factors being released by viable tumor cells in response to the MSV-nAb-PTX system. M1 macrophages are simulated to release nitric oxide, which inhibits cell viability, while M2 macrophages release tumor growth factors, which promote cellular proliferation (5). Each macrophage acts as a source of drug to simulate the release of PTX from the MSV-nAb-PTX formulation. With MSV-nAb-PTX at $24 \mathrm{~h}$ post single treatment, 

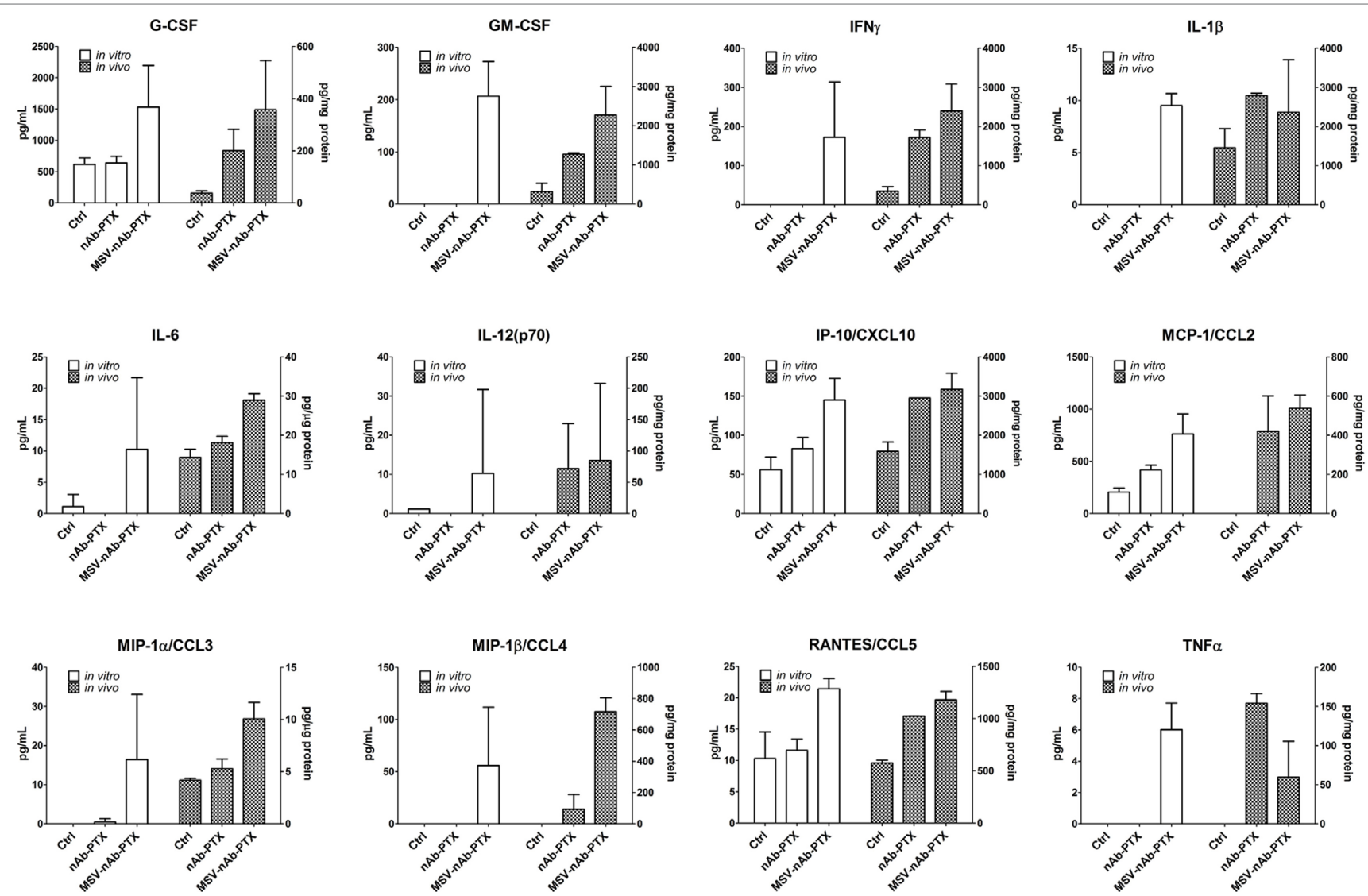

FIGURE 5 | Effect of pretreatment of macrophages with multistage nanovectors (MSV)-albumin-bound paclitaxel (nAb-PTX) vs. nAb-PTX and control of cytokine production by the tumors in vitro and in vivo. Cytokine release was analyzed using MILLIPLEX MAP Mouse Cytokine/Chemokine Immunology Multiplex Assay (EMD Millipore, Billerica, MA, USA) and measured by Luminex $200^{\text {TM }}$ (Luminex, Austin, TX, USA). For in vitro evaluation, the systems were preincubated with macrophages and the conditioned media were introduced to the tumor spheres and incubated for 2 days. For the in vivo study, liver metastatic lesions as well as the surrounding area of the lesion (tumor microenvironment) were dissected and processed for analysis as described in Section "Materials and Methods."

the tumor has slightly shrunk (top right) compared to the initial lesion (5\% radius decrease), while the drug is being released by the macrophages. At $72 \mathrm{~h}$, the lesion radius attains the highest regression (68\% of its original size), by which time most of the drug has been released from the surrounding macrophages. These results are consistent with our previous modeling work (25).

Figure 7 compares the relative contribution of the macrophage polarization in conjunction with MSV-delivered drug to the tumor progression over the course of 5 days after a single treatment. As expected, the cases without treatment are projected to grow unbounded, with the M2-only and the M1/M2 cases attaining 157 and $156 \%$ of their original radius, respectively, while the case without M1/M2 or M1-only, respectively, reaches 143 or $138 \%$. In contrast, all of the MSV-nAb-PTX-treated cases experience regression, which is modulated by the contribution of the macrophage differentiation. The most therapeutically effective scenario is the case with M1-only, reaching $83 \%$ of the original radius, followed by the case with both M1 and M2 present, attaining $94 \%$. The cases with M2-only and without any macrophages are anticipated to reach 118 and $111 \%$ of their original radius, respectively. Interestingly, the model projects that the presence of the M2 phenotype enhances drug cytotoxicity due to the M2 tumor growth-promoting effect enlarging the subset of the tumor population that is susceptible to the cell cycle-specific activity of PTX. However, over the long term, the cases with M2 macrophages recover faster than the cases without their presence, thus promoting tumor growth.

\section{DISCUSSION}

It is currently well recognized that the fine interplay between deregulation of tumor cells and the cells of the TME is imperative for all stages of tumor development (27). Macrophages represent the major population of infiltrating immune cells in TME (28). Macrophage polarization is detrimental in the development and progression of cancer (28). TAMs generally belong to the subclass of alternatively differentiated, M2-like macrophages. They have been shown to modulate tissue remodeling and angiogenesis, suppress $\mathrm{T}$ cell proliferation, and play a significant role in tumor survival (5). High M2 macrophage density has been clinically correlated with poor prognosis in several epithelial cancers, including breast cancer (29) and hepatocellular carcinoma (30). 


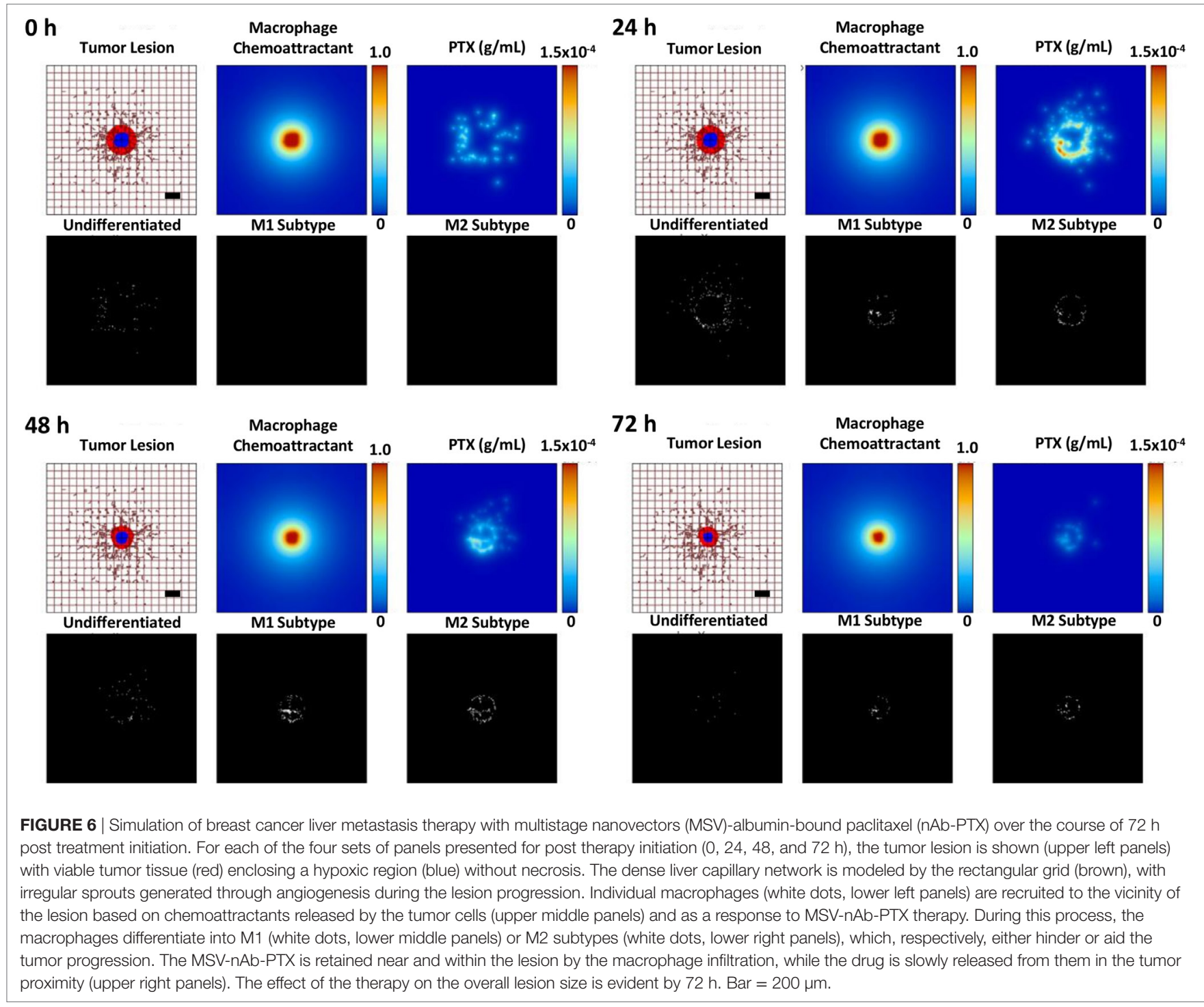

On the other hand, clinical studies have shown that an increased $\mathrm{M} 1 / \mathrm{M} 2$ ratio in the TME is linked to extended survival in ovarian (31), gastric (10), colorectal (32), and lung (33) tumors. M2-like TAMs are characterized by a constitutive high expression of multiple tumor growth promoting factors, including VEGF, FGF1 and 2, PDGF, GM-CSF, insulin-like growth factor-1, and TGF- $\beta$ (34). For example, in a mouse model of breast cancer, expression of CSF-1 was highest at the invasive edge of the malignancy, which was consequently enriched with M2 macrophages. Epidermal growth factor released by these macrophages increased tumor cell migration and metastasis (35). Flexibility and plasticity represent the key characteristics of the cells of mononuclear phagocytic system and their activation states $(5,36)$ Polarization of the macrophages between the M1 and M2 general subtypes can be reversed, as was shown in in vitro and in vivo studies (37). Pathological changes in inflammatory states can sculpture this transition, with M1 macrophages presentatinitiation and during progression of the inflammatory process and M2 macrophages participating in its resolution. In cancer, histidine-rich glycoprotein (a host-produced protein deposited in the stroma) was shown to induce TAM reprogramming from $\mathrm{M} 2$ to $\mathrm{M} 1$, resulting in vascular normalization and improved response to chemotherapy (38).

Our previous study also identified the enrichment of macrophages in the tumor periphery of breast cancer liver metastases in a mouse model (24). We have shown that by directing transport of an Ab-bound drug, nAb-PTX, toward the macrophages in the tumor periphery in the liver using MSV, we could increase the concentration of the drug in the lesions and, consequently, the tumor killing efficiency. However, the pronounced anti-tumor effect observed with MSV-nAb-PTX in this study could not be fully explained only by the shift of the concentration of the drug toward the tumor lesions; thus, in the present work we aimed to evaluate the effect of MSV-nAb-PTX on the inflammatory state of the TME, the migratory potential of the macrophages in the tumor lesion and on the interactions of macrophages with the tumor cells. The studies were performed in vitro in a previously 


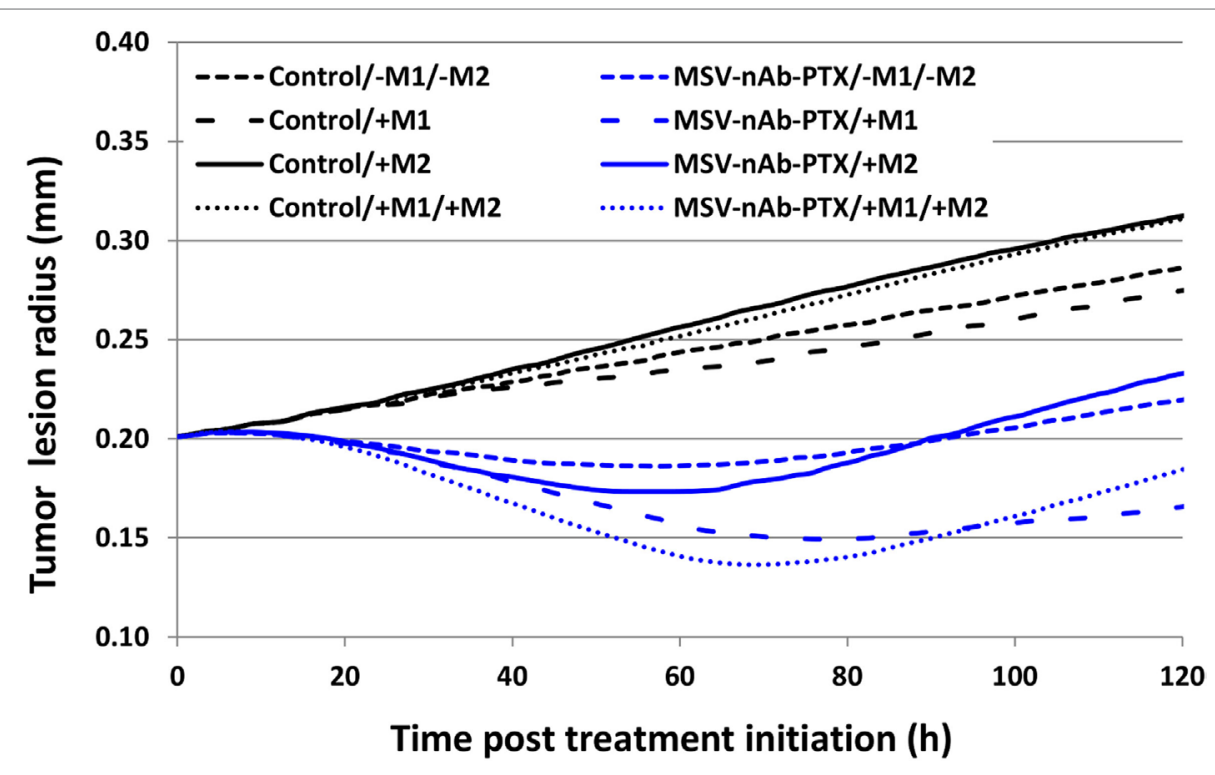

FIGURE 7 | Simulation of tumor progression for untreated and multistage nanovectors (MSV)-albumin-bound paclitaxel (nAb-PTX)-treated cases including various combinations of macrophage polarizations simulated over the course of 5 days post treatment initiation. Control: untreated tumors; MSV-nAb-PTX: tumors treated with the therapeutic system. M1 and M2 refer to the addition of the polarized macrophage populations to the model. The cytotoxic effect of the M1 subtypes is simulated to affect the tissue proportional to the concentration of nitrous oxide released in the immediate vicinity of the macrophage. M2 macrophages release diffusible growth factors which promote tumor proliferation. In addition, treated macrophages (M1 and M2) are simulated to release drug which only affects proliferating tissue due to the cell cycle-dependent effect of PTX.

validated 3D model of hypovascularized breast cancer lesions with macrophages on the lesion periphery $(25,39)$.

Tumor cell proliferation and apoptosis analysis (Figure 1) confirmed that MSV-nAb-PTX preincubated with macrophages had a pronounced therapeutic efficacy, in line with the in vivo data (24). It is important to note that in this experimental set we did not expose the tumor spheres to the drugs directly, but only to the macrophages preincubated with the systems, similar to the in vivo situation, where hypovascularized breast cancer lesions in the liver are surrounded by macrophages. Preincubation of macrophages with rapamycin, an mTOR inhibitor that is known to induce the polarization of macrophages toward the M1 phenotype (26), had a mild effect on tumor cell proliferation, but significantly increased the number of apoptotic cells in the lesions; thus showing that M1 polarization induced tumor cell apoptosis.

Furthermore, we have analyzed the number of M1 and M2 polarized macrophages in the tumor lesions and the localization of the macrophages within the tumor cores in vitro and in vivo (Figures 2-4). As expected, the control (untreated) tumors had increased population of alternatively activated M2-like macrophages. The M2 phenotype is characterized by an improved phagocytic activity (40), since this general subcategory of macrophagesfightsinflammation and participatesin tissue remodeling. M2 macrophages uptake solid particles more efficiently, which helps to concentrate nAb-PTX delivered through MSV. In vitro, all treatments shifted this ratio to a new homeostasis, increasing the population of M1 macrophages and decreasing the fraction of M2 macrophages (Figure 2). With MSV-nAb-PTX, this effect was the most prominent, and the population of M1 activated macrophages increased 20-fold while M2 macrophages represented less than $5 \%$ of the total number of macrophages. Treatment with nAb-PTX in vitro yielded equal populations of M1 and M2 macrophages. Although M1 macrophages predominated with rapamycin, the overall number of macrophages decreased due to a toxic effect of the drug related to inhibition of the mTOR pathway, which is in line with the reported mechanism of rapamycin to induce apoptotic cell death in M0/M2 but not M1 macrophages (26). Interestingly, only MSV-nAb-PTX, but not nAb-PTX caused the shift in the macrophage polarization state in vivo. This could be due to the longer retention of the MSV-nAb-PTX in the lesion and specific association of the carrier with the macrophages (24). PTX has been reported to possess a lipopolysaccharide (LPS)-like property, activating murine macrophages by mimicking bacterial LPS through binding to MD2, an extracellular protein of TLR4 (41). LPS-dependent TLR4 can be activated by PTX and internalized into endosomes, activating downstream signaling pathways via endocytic shuttling, and therefore promoting polarization of macrophages toward the M1 phenotype. A recent study demonstrated the ability of nAb-PTX to enhance the macrophage activation process due to macropinocytic uptake and the fusing of macropinosomes and endosomes (42). In our study, the increased concentration of PTX in the TME mediated by MSV-nAb-PTX induced the release of pro-inflammatory cytokines by the tumor cells, promoting the pro-inflammatory milieu in the TME and modulating the macrophages to undergo M2 to M1 polarization. Our results also suggest that the Ab component of the nAb-PTX may be involved in this process and slightly increased macrophage migration toward the center of the tumor spheroids (Figure 4), although further study is needed for deeper understanding. Ab has been previously reported to contribute to the intratumoral 
concentration increase of nAb-PTX via binding to the $60 \mathrm{kDa}$ glycoprotein receptor and thus increasing transcytosis (43).

We extended the computational model presented in our previous study by Leonard et al (25) to account for macrophage polarization into M1 and M2 subtypes. The simulations provide a platform to analyze the respective effects of different subsets of macrophages in the tumor in combination with MSV-nAb-PTX therapy with the ultimate goal to optimize treatment outcomes. The modeling results suggest that a single therapy may delay the tumor growth in vivo but not completely eradicate the lesion. One reason is that insufficient drug is released by the macrophages in the tumor vicinity to kill all of the tumor cells. Modulation of the macrophage population to increase its size and further drive its polarization toward tumorigenicity, e.g., with an immunotherapy, may achieve a stronger one-time response. However, as shown in our previous study, repeated treatments at regular intervals may still be necessary for complete remission to account for the time it takes for hypoxic (quiescent) cells to resume cycling and thus be sensitive to the chemotherapeutic. We further note that the simulations reflect the variability in experimental measurements regarding the effect of the macrophages. The untreated case with no macrophages and the untreated case with both subtypes could be more similar than shown in Section "Results" (Figure 7), while the effect of the M1 macrophages was calibrated to the low end of possible values. Adjusting for these factors, however, does not affect the overall response difference predicted between untreated and treated cases or the response-modulating effect projected for the M2 macrophages.

Various effects can contribute to the increased efficacy of MSVnAb-PTX via inflammatory modulation. Overall, MSV-nAbPTX increased the motility and directionality of the macrophages toward the tumor sphere (Figure 3). The increased macrophage recruitment may be a response to the increased chemokine release by tumor cells such as CXCL-10, CCL-2, CCL3, CCL4, and CCL5 (Figure 5). Furthermore, the treatment caused deeper macrophage penetration inside the spheroid/tumor lesions (Figure 4), which can correspond with the apoptotic feedback between the dying tumor cells (Figure 1) and the macrophages bearing MSV-nAb-PTX. It is interesting to note that in the therapeutic concentration tested, macrophage polarization (Figure S1 in Supplementary Material) and viability $(24,25)$ were not affected by the treatment with any of the tested systems.

In hypovascularized lesions in the liver, tumor cells are not directly exposed to the circulating drugs, while the macrophages present in the liver vasculature are exposed to the intravenously administered drug/particles. To mimic this situation, we further incubated the macrophages with nAb-PTX and MSV-nAb-PTX for $1 \mathrm{~h}$ [in clinic, $90 \%$ of $\mathrm{nAb}-\mathrm{PTX}$ is cleared from the circulation in this time frame (44)] and further let the macrophages release soluble factors and the internalized drug, exposing the tumor cells to supernatants from the pretreated macrophages (conditioned media collected at $24 \mathrm{~h}$ past drug removal). Cytokine and chemokine profiles were also analyzed in the murine model of liver metastasis. In both the in vitro tumor spheres and the in vivo murine model, there was a significant increase in the factors associated with M1 macrophage polarization, such as CCR5-binding chemokines (CCL3, CCL4, and CCL5) (45), interleukins (IL-6 and IL-1 $\beta$ ), and TNF- $\alpha$ (46). A significant increase in GM-CSF levels released by the tumors in vitro and in vivo in response to exposure to macrophages preincubated with MSV-nAb-PTX can impart an additional feedback on the M1 polarization state (47), as exposure to GM-CSF was previously shown to promote M1 polarization of the macrophages $(47,48)$.

These findings could explain the attraction of the macrophages toward the tumor spheres in vitro and into the tumor core in vitro and in vivo. In contrast to previous studies showing that cancer cell apoptosis shifted the phenotype of macrophages toward M2 $(49,50)$, we have observed that the effects of MSV-nAb-PTX enhanced apoptosis of tumor cells. This can be explained by the fact that there is a direct effect of the system on the macrophage polarization toward the M1 phenotype. Activated M1 macrophages have been recently reported to produce and excrete chitotriosidases (or family 18 chitinases), which can modulate proteases and cause damage to cancer cell membranes (51).

In conclusion, our data demonstrate that macrophages carried MSV-nAb-PTX while not being affected by the therapeutics. The phagocytosis of the solid particles by the macrophages enhanced the drug concentrations inside of these immune system cells (24), consequently enhancing concentrations of the drug released by macrophages in the TME. As a result, tumor cells were exposed to higher concentrations of drug, resulting in enhanced tumor-cell killing, while also inducing an LPS-like effect of PTX as described by Byrd-Leifer et al. (52). This prompted tumor cells to release higher levels of pro-inflammatory cytokines, causing further shift of macrophage polarization toward the anti-tumorigenic M1 phenotype. The data also suggest that polarization of the nanovectors contributes to the toxicity toward cancer cells. Altogether, these phenomena could be utilized to design improved nanovectorbased cancer therapies.

\section{MATERIALS AND METHODS}

\section{Fabrication of nAb-PTX-MSV}

Albumin-bound paclitaxel-multistage nanovectors- or fluorescently labeled Ab loaded, Ab-MSV, were fabricated and characterized as previously described $(24,25)$. Briefly, MSV with $1 \times 0.4 \mu \mathrm{m}(d \times h)$ dimensions were fabricated in a microelectronics facility via photolithography and electrochemical etching and further oxidized with 3-aminopropyl-triethoxysilane (APTES) $(24,53)$. APTES-MSV were lyophilized using the Freezone Freeze Dry System (Labconco, Kansas City, MO, USA). nAb-PTX (Abraxane $^{\circledR}$, Celgene, Summit, NJ, USA) or Ab (Ab-fluorescein isothiocyanate conjugate, Sigma-Aldrich, St. Louis, MO, USA) concentrated solution was loaded to dried MSV particles in aliquots. Loading was enhanced by a drying process via incubation of the particles under low pressure $(25,54)$. nAb-PTX-MSV was characterized for morphology, zeta potential, and the loading efficiency as described earlier $(24,25)$.

\section{Cell Culture}

Breast cancer 4T1 cells (ATCC, Manassas, VA, USA) were cultured in Minimum Essential Medium (MEM) with 10\% FBS, $1 \%$ antibiotic/antimycotic, $1 \%$ GlutaMAX, $1 \%$ NEAA, $1 \%$ MEM vitamin, and $1 \%$ sodium pyruvate supplements and maintained in humidified atmosphere at $37^{\circ} \mathrm{C}$ and $5 \% \mathrm{CO}_{2}$. Mice macrophages 
were obtained by isolation from fresh mice bone marrow. Monocytes were washed twice with PBS and erythrocytes were lysed by red cell lysis buffer (Sigma, USA), and cells were filtered with a $70 \mu \mathrm{m}$ filter (BD Lifesciences, USA). Differentiation of monocytes to resting macrophages was initiated by 7-day incubation with macrophage medium, containing 10\% FBS and 1\% penicillin/streptomycin in RPMI 1640 medium.

\section{D TME Model: Coculture of Breast Cancer Spheres and Macrophages}

Tumor spheres were generated using the Bio-Assembler ${ }^{\mathrm{TM}}$ system based on protocols we recently reported $(39,55)$ and grown to $\sim 450-500 \mu \mathrm{m}$ diameter before cytotoxicity and migration studies. Depending on the studies, macrophages were treated with rapamycin, Ab, MSV-Ab, nAb-PTX, MSV, or MSV-nAbPTX for $4 \mathrm{~h}$ and stained with Vybrant Cell-Labeling Solutions (Molecular Probes, Eugene, OR, USA). After treatment, supernatants were removed and cells were washed with fresh medium to ensure that no $4 \mathrm{~T} 1$ cancer cells are not in contact with drugs in the solution (similar to the clinically relevant situation in hypovascularized tumor lesions). Primary macrophages $\left(1 \times 10^{3}\right)$ were cultured together with $4 \mathrm{~T} 1$ spheres in a 96-well plate and kept in an incubator. Images were taken by fluorescent microscopy after 24, 48, 72, and $96 \mathrm{~h}$ and analyzed with NISElements software.

Transwell plates (Corning Inc., Corning, NY, USA) were utilized for coculture study to analyze macrophage differentiation. $4 \mathrm{~T} 1$ cells $\left(1.5 \times 10^{4}\right)$ were seeded on the apical side while $\left(1.5 \times 10^{3}\right)$ macrophages were seeded on the basolateral side. After $96 \mathrm{~h}$, macrophages were harvested and stained for CD80 and CD204.

\section{Cell Staining and Confocal Microscopy}

Cocultures of macrophages and 4T1 spheres were fixed with $4 \%$ paraformaldehyde before staining. Spheres were stained for TUNEL with FITC for apoptosis detection with Promega TUNEL kit (Promega, Madison, WI, USA) according to the manufacturer's protocol. To assess proliferation the samples were incubated overnight with primary rabbit-anti-mouse Ki67 antibody (1:500, Abcam, Cambridge, UK), washed twice with PBS and incubated with Alexa Fluor 647-labeled goat anti-rabbit secondary antibody for $4 \mathrm{~h}$. Samples were washed twice with PBS before further analysis by confocal microscopy.

Rat anti-mouse CD80 (Thermo Scientific, Rockford, IL, USA) and Alexa Fluor 647-labeled CD204 (Abcam, Cambridge, UK) were utilized for surface marker staining of the macrophages in the coculture. After paraformaldehyde fixation, samples were washed twice with PBS and incubated with $1 \%$ BSA for $20 \mathrm{~min}$. CD80 antibody $(5 \mu \mathrm{g} / \mathrm{ml})$ was added and incubated with the samples overnight at $4^{\circ} \mathrm{C}$. After washing with PBS, goat anti-rat FITC-labeled antibody was added to the samples for $2 \mathrm{~h}$ at RT. Furthermore, samples were washed and stained with Alexa Fluor 647-labeled anti-CD204 (2 h, RT). Prior to confocal microscopy analysis, the samples were washed twice with PBS.

Tumor spheres and macrophages were visualized using a Nikon A1 confocal microscope (Nikon Inc., Melville, NY, USA) based on the fluorescence of the respective probes and analyzed with NIS elements software (Nikon Inc.). Macrophage signal intensity, quantification of macrophages of various phenotypes, and macrophage penetration into the tumor lesion were assessed as below.

\section{Tracking of Macrophage Migration Kinetics, Directionality, and Dynamics As a Function of Treatments}

For the tracking of macrophage migration toward the tumor spheres, the cells were stained with DiD membrane dye (Invitrogen, USA), pretreated with nAb-PTX, MSV, or MSV-nAb-PTX for $1 \mathrm{~h}$ and washed. Furthermore, the macrophages were cocultured with breast cancer spheres as described earlier. To differentiate between the two cell populations, $4 \mathrm{~T} 1$ breast cancer cells were prestained with $1 \mu \mathrm{g} / \mathrm{mL}$ Hoechst 33342 dye (Thermo Scientific). Tumor spheres and macrophage movements were tracked using a live-imaging system Nikon TiEclipse fluorescence microscope (Nikon Inc., USA) over the time course of $10 \mathrm{~h}$ and analyzed with NIS elements. The motility of 4T1 spheres was recorded over time and used as the reference for macrophage displacement calculation. Macrophages speed, path length, as well as coordinates were tracked using NIS elements and calculated for their directionality toward 4T1 spheres using the initial coordinates of the cell vs. the tumor sphere as a reference point.

\section{MTT Assay}

3-(4,5-Dimethylthiazol-2-yl)-2,5-diphenyltetrazolium bromide (Sigma, USA) assay was performed to access cell viability. 4T1 spheres in coculture with macrophages were seeded on 96-well plates before treatment. After 48 or $96 \mathrm{~h}$ of incubation, the cells were washed twice with PBS and the MTT assay was run based on the manufacturer instructions. The absorbance was determined using a spectrophotometer (Biotek, Winooski, VT, USA) at $570 \mathrm{~nm}$.

\section{In Vivo Model of Breast Cancer Liver Metastasis}

Animal studies were performed in accordance with approved protocols by Houston Methodist Research Institute Institutional Animal Care and Use Committee (AUP-0514-0032). Balb/c mice were purchased from Charles Rivers Laboratories and mouse breast cancer liver metastases xenograft were generated by splenic injection of $10^{5} 4 \mathrm{~T} 1$ tumor cells/100 $\mu \mathrm{L}$ PBS as we previously described $(24,43)$. Splenectomy was conducted immediately after injection to prevent primary tumor growth in the spleen, and the xenografts were grown for at least 10 days before therapy.

\section{In Vivo Evaluation of Macrophage Quantity within the Microenvironment}

For analysis of TME changes in response to therapy in vivo, mice with cancer liver metastasis were randomly divided into three groups $(n=4)$ : control, nAb-PTX, MSV-nAb-PTX-nAb-PTX, and MSV-nAb-PTX containing $75 \mathrm{mg} / \mathrm{kg} \mathrm{nAb}-\mathrm{PTX}(7.5 \mathrm{mg} / \mathrm{kg}$ PTX) were injected via the tail vein. The treatment was repeated every 3 days and the mice were sacrificed after three treatments. 
The liver was dissected, embedded in OCT compound (Sakura ${ }^{\circledR}$ Finetek USA, Inc., Torrance, CA, USA), and cut in $4 \mu \mathrm{m}$ sections for histological and immunofluorescence analyses. The frozen sections were fixed with ice-cold acetone and stained with Alexa Fluor 488-tagged rat anti-mouse F4/80 antibody and TRITC anti-mouse CD204 antibody to detect total macrophages and the M2 subpopulation, respectively. We have used the CD204 marker to characterize alternatively polarized M2 macrophages $(56,57)$ and F4/80 as a marker for general population of macrophages. Cell nuclei were stained with 4,6-diamidino-2-phenylindole, dihydrochloride.

\section{Cytokines Analysis}

For in vitro cytokine and chemokine analysis, macrophages were plated in a 96-well plate, with a density of 10,000 cells/well and treated with $150 \mathrm{ng}$ nAb-PTX or MSV-nAb-PTX for $1 \mathrm{~h}$. Drug treatment was removed, cells were washed twice with PBS, and fresh medium was added to the macrophages. This was performed to mimic the clinically relevant situation, as clinical studies with nAb-PTX revealed that more than $90 \%$ of the drug is cleared from circulation within $1 \mathrm{~h}$ following intravenous administration (44). Supernatants (conditioned media) were harvested from macrophages after $24 \mathrm{~h}$ and $50 \mu \mathrm{L}$ of this conditioned media were added to $50 \mu \mathrm{L}$ fresh media to culture preformed tumor spheres. Supernatants from 4T1 spheres were harvested after 2 days, and the cytokine and chemokine release was analyzed by MILLIPLEX MAP Mouse Cytokine/Chemokine Immunology Multiplex Assay (EMD Millipore, Billerica, MA, USA) and measured by Luminex $200^{\mathrm{TM}}$ (Luminex, Austin, TX, USA). Additionally, to determine the effect of treatments to the macrophages themselves, the treated macrophages were further cultured with $100 \mu \mathrm{L}$ fresh medium for 3 days. After incubation, the culture media were collected for a cyto-/chemokine release study.

From the in vivo studies, liver metastatic lesions as well as the surrounding area of the lesion (TME) were dissected. Tissues were weighed, $500 \mu \mathrm{L}$ PBS with $1 \times$ HALTTM protease inhibitor cocktail (Thermo Fisher Scientific, Waltham, MA, USA) was added to the samples, and the samples were homogenized using Polytron PT2100 homogenizer (Kinematica AG, Lucerne, Switzerland). Tissue lysates were incubated under constant agitation for $2 \mathrm{~h}$ and the supernatants were separated by centrifugation at $10,000 \times g$ for $20 \mathrm{~min}$ at $4^{\circ} \mathrm{C}$. Supernatants containing protein extracts were used for cyto-/chemokine measurements. Protein content of the supernatants was determined using Pierce $^{\mathrm{TM}}$ BCA Protein Assay Kit (Thermo Fisher Scientific, Waltham, MA, USA) for normalization of further measurements. Cyto-/chemokines were analyzed by MILLIPLEX MAP Mouse Cytokine/Chemokine Immunology Multiplex Assay (EMD Millipore, Billerica, MA, USA) and measured by Luminex $200^{\mathrm{TM}}$ (Luminex, Austin, TX, USA).

\section{Mathematical Model}

We applied mathematical modeling to computationally simulate the tumor response as a function of MSV-nAb-PTX-coupled macrophages differentiating into M1- and M2 subtypes. As described in our previous work (25), the model (58-61) simulates viable and necrotic tissue in hepatic metastases, including the transport of macrophages and molecules through this tissue. The tumor growth is obtained through balance of cell proliferation and death. Proliferation depends on adequate oxygen and cell nutrients, while death is induced by levels of oxygen below a threshold of viability as well as drug above a certain level of cytotoxicity. Values for the model parameters were calibrated to our experimental data as in Ref. $(25,58-61)$. We simulated release of paclitaxel from $n A b-P T X$ carried by nanovector-loaded macrophages infiltrating the tumor tissue and differentiating into M1- and M2 subtypes. The model and associated parameters are further described in the Supplementary Material.

\section{Statistical Analysis}

All quantitative parameters are presented as mean values with SD. Statistical analysis was performed by $t$-test for unpaired samples using Graphpad Prism software, with $p$-value $<0.05$ accepted as indicative of significant difference, $<0.01$ as a statistically very significant difference.

\section{ETHICS STATEMENT}

Animal studies were performed in accordance with approved protocols by Houston Methodist Research Institute Institutional Animal Care and Use Committee (IACUC) (AUP-0514-0032).

\section{AUTHOR CONTRIBUTIONS}

BG, FL, and HF conceived the idea and designed the research. FL and TN performed in vitro experiments and analyzed the data. FL and MW analyzed the live-cell imaging data. FL and KY performed in vivo studies. LC and HF developed computational models and therapy simulations. XL fabricated MSV. FL, HF, and BG wrote the manuscript. All the authors reviewed and approved the manuscript.

\section{ACKNOWLEDGMENTS}

We would like to thank Megumi Kai and Yan Ting Liu for providing mice bone marrow for monocyte isolation, and Carlotta Borsoi for her assistance with immunostaining of the histological slides from in vivo studies.

\section{FUNDING}

BG acknowledges the support from R21HD08947. BG and XL acknowledge the support from 1R21CA190024-01A1.

\section{SUPPLEMENTARY MATERIAL}

The Supplementary Material for this article can be found online at http://journal.frontiersin.org/article/10.3389/fimmu.2017.00693/ full\#supplementary-material. 


\section{REFERENCES}

1. Balkwill F, Mantovani A. Inflammation and cancer: back to Virchow? Lancet (2001) 357:539-45. doi:10.1016/S0140-6736(00)04046-0

2. Coussens LM, Werb Z. Inflammation and cancer. Nature (2002) 420:860-7. doi:10.1038/nature01322

3. Balkwill F, Charles KA, Mantovani A. Smoldering and polarized inflammation in the initiation and promotion of malignant disease. Cancer Cell (2005) 7:211-7. doi:10.1016/j.ccr.2005.02.013

4. Martinez FO. Regulators of macrophage activation. Eur J Immunol (2011) 41:1531-4. doi:10.1002/eji.201141670

5. Mantovani A, Sozzani S, Locati M, Allavena P, Sica A. Macrophage polarization: tumor-associated macrophages as a paradigm for polarized M2 mononuclear phagocytes. Trends Immunol (2002) 23:549-55. doi:10.1016/S1471-4906(02) 02302-5

6. Jakubzick CV, Randolph GJ, Henson PM. Monocyte differentiation and antigen-presenting functions. Nat Rev Immunol (2017) 17(6):349-62. doi:10.1038/nri.2017.28

7. Sica A, Schioppa T, Mantovani A, Allavena P. Tumour-associated macrophages are a distinct $\mathrm{M} 2$ polarised population promoting tumour progression: potential targets of anti-cancer therapy. Eur J Cancer (2006) 42:717-27. doi:10.1016/j.ejca.2006.01.003

8. Mantovani A, Allavena P, Sica A. Tumour-associated macrophages as a prototypic type II polarised phagocyte population: role in tumour progression. Eur J Cancer (2004) 40:1660-7. doi:10.1016/j.ejca.2004.03.016

9. Cao W, Peters JH, Nieman D, Sharma M, Watson T, Yu J. Macrophage subtype predicts lymph node metastasis in oesophageal adenocarcinoma and promotes cancer cell invasion in vitro. Br J Cancer (2015) 113:738-46. doi:10.1038/bjc.2015.292

10. Pantano F, Berti P, Guida FM, Perrone G, Vincenzi B, Amato MMC, et al. The role of macrophages polarization in predicting prognosis of radically resected gastric cancer patients. J Cell Mol Med (2013) 17:1415-21. doi:10.1111/ jcmm.12109

11. Lan C, Huang X, Lin S, Huang H, Cai Q, Wan T, et al. Expression of M2-polarized macrophages is associated with poor prognosis for advanced epithelial ovarian cancer. Technol Cancer Res Treat (2013) 12:259-67. doi: $10.7785 /$ tcrt.2012.500312

12. Bronkhorst IHG, Ly LV, Jordanova ES, Vrolijk J, Versluis M, Luyten GPM, et al. Detection of M2-macrophages in uveal melanoma and relation with survival. Invest Ophthalmol Vis Sci (2011) 52:643-50. doi:10.1167/iovs.10-5979

13. Chávez-Galán L, Olleros ML, Vesin D, Garcia I. Much more than M1 and M2 macrophages, there are also CD169(+) and TCR(+) macrophages. Front Immunol (2015) 6:263. doi:10.3389/fimmu.2015.00263

14. Goswami KK, Ghosh T, Ghosh S, Sarkar M, Bose A, Baral R. Tumor promoting role of anti-tumor macrophages in tumor microenvironment. Cell Immunol (2017) 316:1-10. doi:10.1016/j.cellimm.2017.04.005

15. Mantovani A, Sica A, Allavena P, Garlanda C, Locati M. Tumor-associated macrophages and the related myeloid-derived suppressor cells as a paradigm of the diversity of macrophage activation. Hum Immunol (2009) 70:325-30. doi:10.1016/j. humimm.2009.02.008

16. Takeya M, Komohara Y. Role of tumor-associated macrophages in human malignancies: friend or foe? Pathol Int (2016) 66:491-505. doi:10.1111/pin.12440

17. Sica A, Mantovani A. Macrophage plasticity and polarization: in vivo veritas. J Clin Invest (2012) 122:787-95. doi:10.1172/JCI59643

18. Stout RD, Jiang C, Matta B, Tietzel I, Watkins SK, Suttles J. Macrophages sequentially change their functional phenotype in response to changes in microenvironmental influences. J Immunol (2005) 175:342-9. doi:10.4049/ jimmunol.175.1.342

19. Georgoudaki A-M, Prokopec KE, Boura VF, Hellqvist E, Sohn S, Östling J, et al. Reprogramming tumor-associated macrophages by antibody targeting inhibits cancer progression and metastasis. Cell Rep (2016) 15:2000-11. doi:10.1016/j.celrep.2016.04.084

20. Olsson A, Nakhlé J, Sundstedt A, Plas P, Bauchet A-L, Pierron V, et al. Tasquinimod triggers an early change in the polarization of tumor associated macrophages in the tumor microenvironment. J Immunother Cancer (2015) 3:53. doi:10.1186/s40425-015-0098-5

21. Jain S, Tran T-H, Amiji M. Macrophage repolarization with targeted alginate nanoparticles containing IL-10 plasmid DNA for the treatment of experimental arthritis.Biomaterials(2015)61:162-77.doi:10.1016/j.biomaterials.2015.05.028
22. Tran T-H, Rastogi R, Shelke J, Amiji MM. Modulation of macrophage functional polarity towards anti-inflammatory phenotype with plasmid DNA delivery in CD44 targeting hyaluronic acid nanoparticles. Sci Rep (2015) 5: 16632. doi:10.1038/srep16632

23. Miao X, Leng X, Zhang Q. The current state of nanoparticle-induced macrophage polarization and reprogramming research. Int J Mol Sci (2017) 18:336. doi:10.3390/ijms18020336

24. Tanei T, Leonard F, Liu X, Alexander JF, Saito Y, Ferrari M, et al. Redirecting transport of nanoparticle albumin-bound paclitaxel to macrophages enhances therapeutic efficacy against liver metastases. Cancer Res (2016) 76:429-39. doi:10.1158/0008-5472.CAN-15-1576

25. Leonard F, Curtis LT, Yesantharao P, Tanei T, Alexander JF, Wu M, et al. Enhanced performance of macrophage-encapsulated nanoparticle albuminbound-paclitaxel in hypo-perfused cancer lesions. Nanoscale (2016) 8: 12544-52. doi:10.1039/c5nr07796f

26. Mercalli A, Calavita I, Dugnani E, Citro A, Cantarelli E, Nano R, et al. Rapamycin unbalances the polarization of human macrophages to M1. Immunology (2013) 140:179-90. doi:10.1111/imm.12126

27. Quail DF, Joyce JA. Microenvironmental regulation of tumor progression and metastasis. Nat Med (2013) 19:1423-37. doi:10.1038/nm.3394

28. Mantovani A, Marchesi F, Malesci A, Laghi L, Allavena P. Tumour-associated macrophages as treatment targets in oncology. Nat Rev Clin Oncol (2017). doi:10.1038/nrclinonc.2016.217

29. Laoui D, Movahedi K, Van Overmeire E, Van den Bossche J, Schouppe E, Mommer C, et al. Tumor-associated macrophages in breast cancer: distinct subsets, distinct functions. Int J Dev Biol (2011) 55:861-7. doi:10.1387/ ijdb.113371dl

30. Zhang QW, Liu L, Gong CY, Shi HS, Zeng YH, Wang XZ, et al. Prognostic significance of tumor-associated macrophages in solid tumor: a meta-analysis of the literature. PLoS One (2012) 7:e50946. doi:10.1371/journal.pone. 0050946

31. Zhang M, He Y, Sun X, Li Q, Wang W, Zhao A, et al. A high M1/M2 ratio of tumor-associated macrophages is associated with extended survival in ovarian cancer patients. J Ovarian Res (2014) 7:19. doi:10.1186/1757-2215-7-19

32. Edin S, Wikberg ML, Dahlin AM, Rutegård J, Öberg Å, Oldenborg P-A, et al. The distribution of macrophages with a M1 or M2 phenotype in relation to prognosis and the molecular characteristics of colorectal cancer. PLoS One (2012) 7:e47045. doi:10.1371/journal.pone.0047045

33. Yuan A, Hsiao YJ, Chen HY, Chen HW, Ho CC, Chen YY, et al. Opposite effects of M1 and M2 macrophage subtypes on lung cancer progression. Sci Rep (2015) 5:14273. doi:10.1038/srep 14273

34. Hanahan D, Coussens L. Accessories to the crime: functions of cells recruited to the tumor microenvironment. Cancer Cell (2012) 21:309-22. doi:10.1016/j. ccr.2012.02.022

35. Wyckoff J, Wang W, Lin EY, Wang Y, Pixley F, Stanley ER, et al. A paracrine loop between tumor cells and macrophages is required for tumor cell migration in mammary tumors. Cancer Res (2004) 64:7022-9. doi:10.1158/0008-5472.CAN-04-1449

36. Biswas SK, Mantovani A. Macrophage plasticity and interaction with lymphocyte subsets: cancer as a paradigm. Nat Immunol (2010) 11:889-96. doi:10.1038/ ni. 1937

37. Guiducci C, Vicari AP, Sangaletti S, Trinchieri G, Colombo MP. Redirecting in vivo elicited tumor infiltrating macrophages and dendritic cells towards tumor rejection. Cancer Res (2005) 65:3437-46. doi:10.1158/0008-5472. CAN-04-4262

38. Rolny C, Mazzone M, Tugues S, Laoui D, Johansson I, Coulon C, et al. HRG inhibits tumor growth and metastasis by inducing macrophage polarization and vessel normalization through downregulation of PlGF. Cancer Cell (2011) 19:31-44. doi:10.1016/j.ccr.2010.11.009

39. Leonard F, Godin B. 3D in vitro model for breast cancer research using magnetic levitation and bioprinting method. Methods Mol Biol (2016) 1406:239-51. doi:10.1007/978-1-4939-3444-7_21

40. Gordon S, Martinez FO. Alternative activation of macrophages: mechanism and functions. Immunity (2010) 32:593-604. doi:10.1016/j.immuni.2010.05.007

41. Zimmer SM, Liu J, Clayton JL, Stephens DS, Snyder JP. Paclitaxel binding to human and murine MD-2. J Biol Chem (2008) 283:27916-26. doi:10.1074/ jbc.M802826200

42. Cullis J, Siolas D, Avanzi A, Barui S, Maitra A, Bar-Sagi D. Macropinocytosis of Nab-paclitaxel drives macrophage activation in pancreatic cancer. Cancer Immunol Res (2017) 5:182-90. doi:10.1158/2326-6066.CIR-16-0125 
43. Desai N, Trieu V, Yao Z, Louie L, Ci S, Yang A, et al. Increased antitumor activity, intratumor paclitaxel concentrations, and endothelial cell transport of cremophor-free, albumin-bound paclitaxel, ABI-007, compared with cremophor-based paclitaxel. Clin Cancer Res (2006) 12:1317-24. doi:10.1158/ 1078-0432.CCR-05-1634

44. Sparreboom A, Scripture CD, Trieu V, Williams PJ, De T, Yang A, et al. Comparative preclinical and clinical pharmacokinetics of a cremophor-free, nanoparticle albumin-bound paclitaxel (abi-007) and paclitaxel formulated in cremophor (Taxol). Clin Cancer Res (2005) 11:4136-43. doi:10.1158/10780432.CCR-04-2291

45. Cassol E, Cassetta L, Rizzi C, Alfano M, Poli G. M1 and M2a polarization of human monocyte-derived macrophages inhibits HIV-1 replication by distinct mechanisms. J Immunol (2009) 182:6237-46. doi:10.4049/jimmunol.0803447

46. Luckett-Chastain L, Calhoun K, Schartz T, Gallucci RM. IL-6 influences the balance between M1 and M2 macrophages in a mouse model of irritant contact dermatitis. J Immunol (2016) 196 (1 Suppl):196.17.

47. Hamilton JA. Rheumatoid arthritis: opposing actions of haemopoietic growth factors and slow-acting anti-rheumatic drugs. Lancet (1993) 342:536-9. doi:10.1016/0140-6736(93)91653-4

48. Fleetwood AJ, Lawrence T, Hamilton JA, Cook AD. Granulocyte-macrophage colony-stimulating factor (CSF) and macrophage CSF-dependent macrophage phenotypes display differences in cytokine profiles and transcription factor activities: implications for CSF blockade in inflammation. J Immunol (2007) 178:5245-52. doi:10.4049/jimmunol.178.8.5245

49. Weigert A, Tzieply N, von Knethen A, Johann AM, Schmidt H, Geisslinger G, et al. Tumor cell apoptosis polarizes macrophages role of sphingosine-1-phosphate. Mol Biol Cell (2007) 18:3810-9. doi:10.1091/mbc.E06-12-1096

50. Ferracini M, Rios FJO, Pecenin M, Jancar S. Clearance of apoptotic cells by macrophages induces regulatory phenotype and involves stimulation of CD36 and platelet-activating factor receptor. Mediators Inflamm (2013) 2013:8. doi:10.1155/2013/950273

51. Pan XQ. The mechanism of the anticancer function of M1 macrophages and their use in the clinic. Chin J Cancer (2012) 31:557-63. doi:10.5732/ cjc.012.10046

52. Byrd-Leifer CA, Block EF, Takeda K, Akira S, Ding A. The role of MyD88 and TLR4 in the LPS-mimetic activity of Taxol. Eur J Immunol (2001) 31:2448-57. doi:10.1002/1521-4141(200108)31:8<2448::AID-IMMU2448> 3.0.CO;2-N

53. Godin B, Chiappini C, Srinivasan S, Alexander JF, Yokoi K, Ferrari M, et al. Discoidal porous silicon particles: fabrication and biodistribution in breast cancer bearing mice. Adv Funct Mater (2012) 22:4225-35. doi:10.1002/ adfm.201290121
54. Leonard F, Margulis-Goshen K, Liu X, Srinivasan S, Magdassi S, Godin B. Low pressure mediated enhancement of nanoparticle and macromolecule loading into porous silicon structures. Mesoporous Biomater (2014) 1. doi:10.2478/ mesbi-2014-0002

55. Jaganathan H, Gage J, Leonard F, Srinivasan S, Souza GR, Dave B, et al. Three-dimensional in vitro co-culture model of breast tumor using magnetic levitation. Sci Rep (2014) 4:6468. doi:10.1038/srep06468

56. Kawamura K, Komohara Y, Takaishi K, Katabuchi H, Takeya M. Detection of M2 macrophages and colony-stimulating factor 1 expression in serous and mucinous ovarian epithelial tumors. Pathol Int (2009) 59:300-5. doi:10.1111/j.1440-1827.2009.02369.x

57. Soldano S, Pizzorni C, Paolino S, Trombetta AC, Montagna P, Brizzolara R, et al. Alternatively activated (M2) macrophage phenotype is inducible by endothelin-1 in cultured human macrophages. PLoS One (2016) 11:e0166433. doi:10.1371/journal.pone.0166433

58. Wu M, Frieboes HB, McDougall SR, Chaplain MAJ, Cristini V, Lowengrub J. The effect of interstitial pressure on tumor growth: coupling with the blood and lymphatic vascular systems. J Theor Biol (2013) 320:131-51. doi:10.1016/j. jtbi.2012.11.031

59. van de Ven AL, Wu M, Lowengrub J, McDougall SR, Chaplain MAJ, Cristini V, et al. Integrated intravital microscopy and mathematical modeling to optimize nanotherapeutics delivery to tumors. AIP $A d v$ (2012) 2:11208. doi:10.1063/ 1.3699060

60. Macklin P, McDougall S, Anderson ARA, Chaplain MAJ, Cristini V, Lowengrub J. Multiscale modelling and nonlinear simulation of vascular tumour growth. J Math Biol (2009) 58:765-98. doi:10.1007/s00285-008-0216-9

61. Wu M, Frieboes HB, Chaplain MAJ, McDougall SR, Cristini V, Lowengrub JS . The effect of interstitial pressure on therapeutic agent transport: coupling with the tumor blood and lymphatic vascular systems. J Theor Biol (2014) 355:194-207. doi:10.1016/j.jtbi.2014.04.012

Conflict of Interest Statement: The authors declare that the research was conducted in the absence of any commercial or financial relationships that could be construed as a potential conflict of interest.

Copyright (c) 2017 Leonard, Curtis, Ware, Nosrat, Liu, Yokoi, Frieboes and Godin. This is an open-access article distributed under the terms of the Creative Commons Attribution License (CC BY). The use, distribution or reproduction in other forums is permitted, provided the original author(s) or licensor are credited and that the original publication in this journal is cited, in accordance with accepted academic practice. No use, distribution or reproduction is permitted which does not comply with these terms. 